\title{
Human Newborn Skin: The Effect of Isopropanol on Skin Surface Hydrophobicity
}

\author{
FELIX A. OKAH, R. RANDALL WICKETT, KATHLEEN POMPA, AND STEVEN B. HOATH \\ Division of Neonatology, Children's Hospital Medical Center [F.A.O., K.P., S.B.H.], College of Pharmacy. \\ University of Cincinnati [R.R.W.J. Cincinnati, Ohio 45267
}

\begin{abstract}
The development of a hydrophobic skin surface in newborn mammals such as the rat plays an important role in promoting adaptation to the abrupt change in the environment that occurs at birth. To determine whether the skin surface plays a similar role in the human neonate, we performed tests of water sorption and desorption on the chest wall of 13 term newborns. These tests were performed within the first $\mathbf{2 4} \mathrm{h}$ of life on unperturbed skin (controls) and after perturbation of a contralateral site with isopropanol. The degree of surface hydration was determined by measurement of skin surface electrical capacitance, and desorption rates were calculated by 1 st-order kinetic analysis. The unperturbed surface of the newborn skin exhibited a peak sorption value (change from baseline after water loading) of $435 \pm 83 \mathrm{pF}$ (mean \pm SEM) and a desorption rate of $0.048 \pm 0.009 \mathrm{~s}^{-1}$. After exposure to isopropanol, the peak sorption value increased to $594 \pm 79$ pF $(p<0.05)$ and the desorption rate decreased to 0.024 $\pm 0.004 \mathrm{~s}^{-1}(p<0.01)$. Paired sorption values were positively correlated $\left(r^{2}=0.8, p<0.001\right)$. These results support the hypothesis that the skin surface of the human newborn, by limiting the sorption of water (or amniotic fluid) on the skin, may play a role in the adaptation to the change in environment at birth. (Pediatr Res 35:443-446, 1994)
\end{abstract}

\section{Abbreviations}

Pc, change in surface capacitance after water loading Kd, desorption rate

During the last trimester, the skin of the human fetus produces increasing amounts of barrier lipids (1-4). These lipids include the intercorneocyte "cement" within the stratum corneum and the outermost lipid layer on the skin surface, which in its most obvious form appears as vernix caseosa. Birth marks a transition for the human newborn from a wet and warm environment to one that is dry and cool. The human newborn is usually aided in this transition by care providers who wipe excess amniotic fluid off the skin. It is important that during this transition the fluid on the surface of the skin is dried off as rapidly as possible to reduce heat loss due to evaporation (5).

In an earlier study, we demonstrated that the hydrophobic surface of the skin plays an important role in the newborn rat in promoting adaptation to the change in environment that occurs at birth (6). Like that of the neonatal rat, the skin surface of the newborn human has been reported to have poor water-binding

Received August 5, 1993; accepted November 15, 1993.

Correspondence and reprint requests: Steven B. Hoath, M.D. Division of Neonatology, Department of Pediatrics, University of Cincinnati Medical Center. 231 Bethesda Ave., Cincinnati, OH 45267 properties (7). When in contact with water, for example, the skin surface of the newborn adsorbs (holds on to) less water than the surface of human adult skin (7). The sources of the hydrophobicity of newborn skin are as yet undetermined but likely include a superficial layer of lipids associated with the vernix caseosa.

In this study, we have focused on determining the degree of water binding of the human newborn skin surface during the first $24 \mathrm{~h}$ of life. The sorption (binding) and desorption (release) of water were measured by electrical capacitance, a standard method for determining the degree of surface hydration (8). Specifically, we measured the peak sorption and the rate of desorption of water on the surface of the skin of 13 term newborns before and after perturbation of the surface with isopropanol. Isopropanol was chosen because it is an organic solvent commonly used in most newborn nurseries as a surface disinfectant and as a "degreasing" agent before the application of adhesive tape and surface electrodes.

\section{MATERIALS AND METHODS}

Human subjects. The study group consisted of 13 term newborns admitted to the Newborn Nursery of the University of Cincinnati Medical Center. All infants were screened by obstetric and family history and physical examination. Exclusion criteria included chorioamnionitis, congenital skin anomalies, maternal drug use of medications, and local abrasions of the skin surface. The infants had an average birth weight of $3200 \pm 52$ (SEM) and an average gestational age of $39 \pm 1 \mathrm{wk}$. Seven of 13 infants were female and eight of 13 were black (five were white), and three infants were delivered by cesarean section. All infants were bathed at $4 \mathrm{~h}$ of age with Safeguard bar soap. The research protocol was approved by the Institutional Review Board of the University of Cincinnati Medical Center and informed consent was obtained from the mothers before enrollment of the infants in the study.

Capacitance measurements. Skin surface capacitance, as a measure of surface hydration, was determined using a batteryoperated Dermaphase Meter, model 9003 (NOVA Technology Corp., Gloucester, MA). This instrument has a spring-loaded probe head with a flat contact surface (diameter $=5 \mathrm{~mm}$ ) containing two concentric brass electrodes separated by a nonconducting resin. The instrument readings (c) were converted to $\mathrm{pF}$ with the formula $\left(8.38 \times 10^{-6} \times \mathrm{c}^{3}\right)-\left(4.0 \times 10^{-3} \times \mathrm{c}^{2}\right)+$ $1.668 \mathrm{c}-122.1$. This formula was generated by fitting a cubic polynomial to the calibration curve provided by NOVA Technology Corp. The capacitance of the skin surface (the ratio of charge to potential on an electrically charged isolated conductor) is directly related to its degree of hydration (8). Capacitance measurements were performed at the bedside and data downloaded on-line to a Compaq laptop computer. Data files were subsequently transferred to a Hewlett-Packard $486 / 66 \mathrm{~N}$ computer for analysis using Lotus 1-2-3 software.

Clinical protocol. Tests of water sorption and desorption were performed in 13 term newborn infants between 12 and $24 \mathrm{~h}$ of 
age. The timing of the study was chosen to conform to the conditions under which other investigators have previously studied the surface of the newborn skin $(7,9)$. Specifically, surface capacitance was measured before (baseline) and after application of water to the skin over the left or right chest wall $1 \mathrm{~cm}$ medial to the nipples. These sites were chosen because the flat topography over the chest allows easy access in newborns lying supine. After baseline capacitance measurements, the control site was wet with $0.5 \mathrm{~mL}$ of water for $10 \mathrm{~s}$, after which excess water on the surface of the skin was blotted three times in succession over a period of $5 \mathrm{~s}$ with sterile cotton gauze (Kendall Healthcare Products Co., Mansfield, MA). Surface capacitance was measured $5 \mathrm{~s}$ after completion of blotting (peak capacitance). After the peak capacitance measurement, subsequent measurements were made at 20-s intervals until capacitance readings returned to baseline. A schema of the sorption-desorption test used is shown in Figure 1.

To determine the effect of perturbing the superficial lipid layer with a common organic solvent, the following procedure was performed: After baseline capacitance measurements, the alternate (test) site was cleaned with three firm strokes of two-ply medium $(3.25 \times 3.7 \mathrm{~cm})$ wipes saturated with $70 \%$ isopropanol (Kendall Healthcare Products Co.) and allowed to dry (until capacitance readings returned to baseline). Subsequently, tests of sorption and desorption were performed as described for control sites with each infant serving as his own control. The environmental temperature and relative humidity in the nursery were 24 to $25^{\circ} \mathrm{C}$ and 38 to $40 \%$, respectively.

Analysis of data. The two primary variables for statistical comparison were the maximum change in capacitance after water loading $(\mathrm{Pc})$ and the desorption rate $(\mathrm{Kd})$. Pc, a measure of water sorption, was determined by subtracting the baseline measurement from the peak capacitance value after loading of the skin surface with water. The $\mathrm{Kd}$ was determined as the slope of the regression line of best fit after logarithmic conversion of the individual data points. This analysis assumes 1st-order kinetics and is modeled by the following equation: $\mathrm{Kd}=\log (\mathrm{Cp}) /$ $\log (\mathrm{Ct}) \times 2.303 / \mathrm{t}$, where $\mathrm{Cp}$ and $\mathrm{Ct}$ are the capacitance measurements at the onset of desorption and at subsequent time $t$.

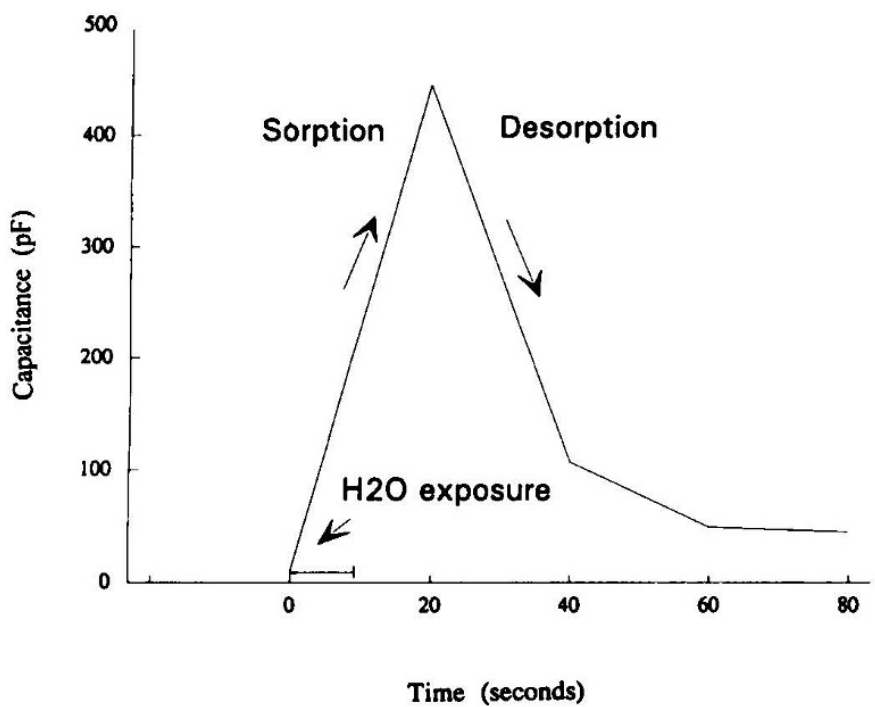

Fig. 1. Schema of the water sorption and desorption test. The ability of the surface of the skin to sorb and desorb water was assessed by a modification of the standard test procedure described by Saijo and Tagami (7). After a baseline reading, the test site was exposed to $0.5 \mathrm{~mL}$ of water for $10 \mathrm{~s}$. Excess water on the skin was removed by blotting with a cotton gauze sponge over a 5-s period. Initial readings were taken $5 \mathrm{~s}$ after blotting, and serial capacitance measurements were taken every 20 $\mathbf{s}$ thereafter. Data were plotted using the onset of water exposure as time 0 .
This formula was also used in calculating the time from mean peak to mean baseline capacitance. The data were analyzed using $t$ test for paired samples and the Wilcoxon signed rank test. A $p$ value less than 0.05 was considered significant. Linear regression analyses were also performed on the individual paired data points to test for correlation within the groups. To graphically illustrate the effect of isopropanol on skin-surface water binding, representative desorption plots were constructed from the composite data using the mean peak sorption (Pc) and the mean $\mathrm{Kd}$ for all 13 newborns. The data were also analyzed for possible differences due to sex and race. All values are expressed as mean \pm SEM.

\section{RESULTS}

Baseline capacitance measurements in 13 term newborn infants at control and test sites were $10 \pm 1.6$ and $7 \pm 1.2 \mathrm{pF}$, respectively. These values did not differ significantly from each other. After tests of water sorption and desorption, control sites exhibited a mean $\mathrm{Pc}$ of $435 \pm 83 \mathrm{pF}$ and a mean rate of desorption of $0.048 \pm 0.009 \mathrm{~s}^{-1}$. When the surface was perturbed with isopropanol, mean sorption increased to $594 \pm 79 \mathrm{pF}(p<$ $0.05)$ and the rate of desorption decreased to $0.024 \pm 0.004 \mathrm{~s}^{-1}$ $(p<0.01)$. Figures 2 and 3 are composite graphic representations of the sorption-desorption profile and the desorption slope after logarithmic transformation of the derived data points for all 13 newborns. Linear regression analysis of water sorption at control and isopropanol-treated sites revealed a significant and positive correlation $(r=0.9, p<0.001)$ (Fig. $4 A$ ). Similar analysis showed a similar but less significant correlation between paired desorption rates at control and isopropanol-treated sites $(r=0.6, p<$ 0.05 ) (Fig. $4 B$ ). The calculated mean time of desorption from peak capacitance to baseline was significantly longer after treatment with isopropanol ( 79 versus $186 \mathrm{~s}, p<0.01$ ). Race and sex were not significant influences. It was not possible to independently evaluate the effect of the mode of delivery on the interaction between the skin surface and exogenous water because of the small number of infants delivered by cesarean section.

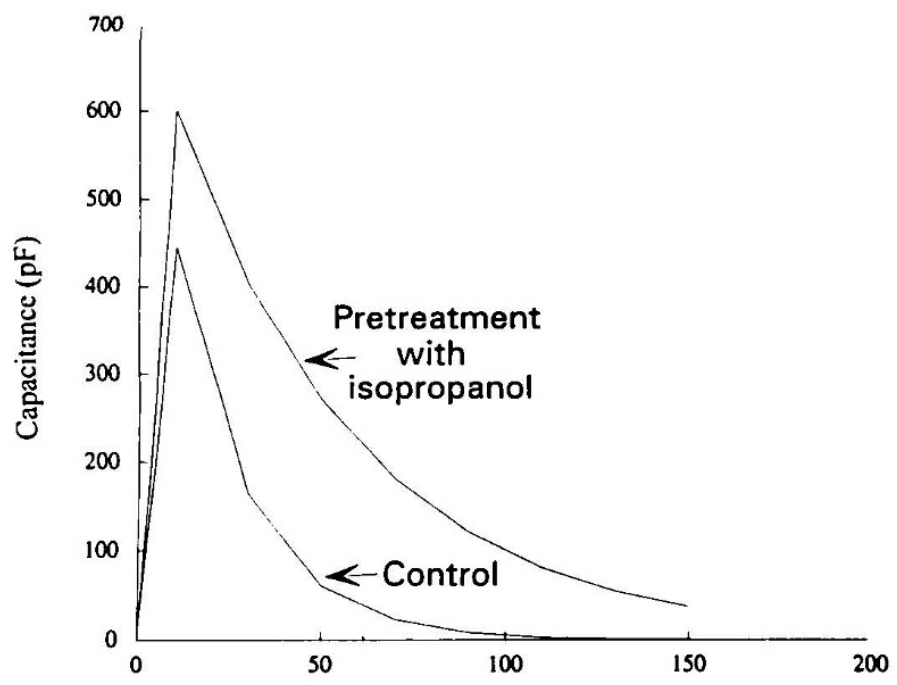

Time (seconds)

Fig. 2. Representative water sorption-desorption profile for the skin (chest) of 13 term newborns and the effect of exposure to isopropanol. The plots represent a composite of data obtained over control and contralateral test sites (see Materials and Methods). Pretreatment with isopropanol resulted in significantly higher peak capacitance $(p<0.05)$ after wetting the skin surface with $0.5 \mathrm{~mL}$ of water. 


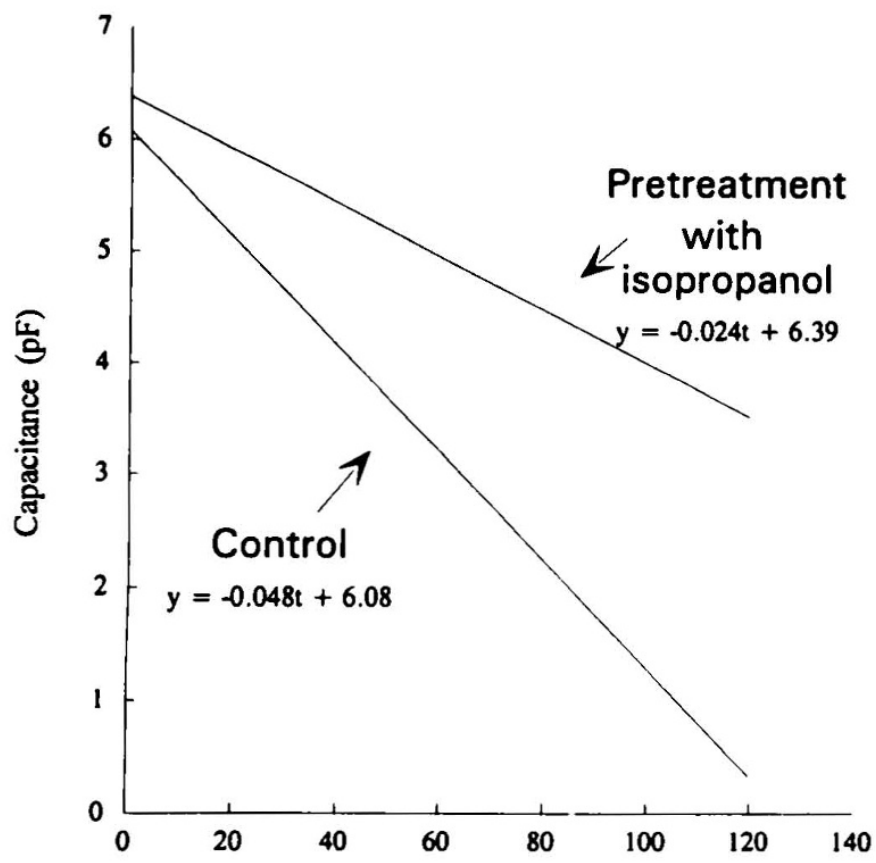

Time (seconds)

Fig. 3. Representative water desorption profiles for newborn skin after logarithmic transformation of capacitance measurements and application of 1st-order kinetic analysis (see Materials and Methods). Pretreatment with isopropyl alcohol resulted in a significant decrease in the calculated desorption rate $(p<0.01)$ after wetting the skin surface with water.

\section{DISCUSSION}

In this study, we provide evidence that the superficial lipid layer of the newborn skin influences the interaction between the skin surface and exogenous water. Tests of sorption-desorption show that when the skin surface is perturbed by exposure to an organic solvent, the skin sorbs more water and subsequently desorbs it at a slower rate. These findings support the hypothesis that a superficial lipid layer confers two related properties on the skin surface, namely, the ability to limit sorption and the ability to expedite the desorption of water. In other words, the skin surface of the newborn is rendered more hydrophobic by the superficial lipid layer.

The barrier lipids of the newborn epidermis are constituted in part by the intercorneocyte lamellar lipids of the stratum corneum and the lipid components of the vernix caseosa (1-4). The vernix, because of its more superficial location, is particularly susceptible to the action of exogenously applied solvents and, hence, is of potential importance in explaining our results. Biochemically, the vernix is a complex, lipid-rich layer that contains large amounts of squalene and cholesterol esters because of the progressive increase in the activity of the sebaceous glands during the last trimester of gestation $(1,3,9)$. Sebaceous gland hypertrophy (commonly seen as milia in the newborn) is generally thought to be secondary to increasing androgenic stimulation by the adrenal glands, which reaches a peak near the time of birth. After birth, the activity of the sebaceous glands decreases abruptly only to increase again between 6 and 8 y of age (1). This sequence of activity is reflected in the compositional study of skin surface lipids in newborns, older children, and adults by Ramasastry et al. (10). These workers found that the skin surface lipids of newborns on the 5th $\mathrm{d}$ of life, despite regular baths, were primarily sebaceous in origin and differed from those of older children and adults.

In addition to biochemical analysis, various electrical meas-
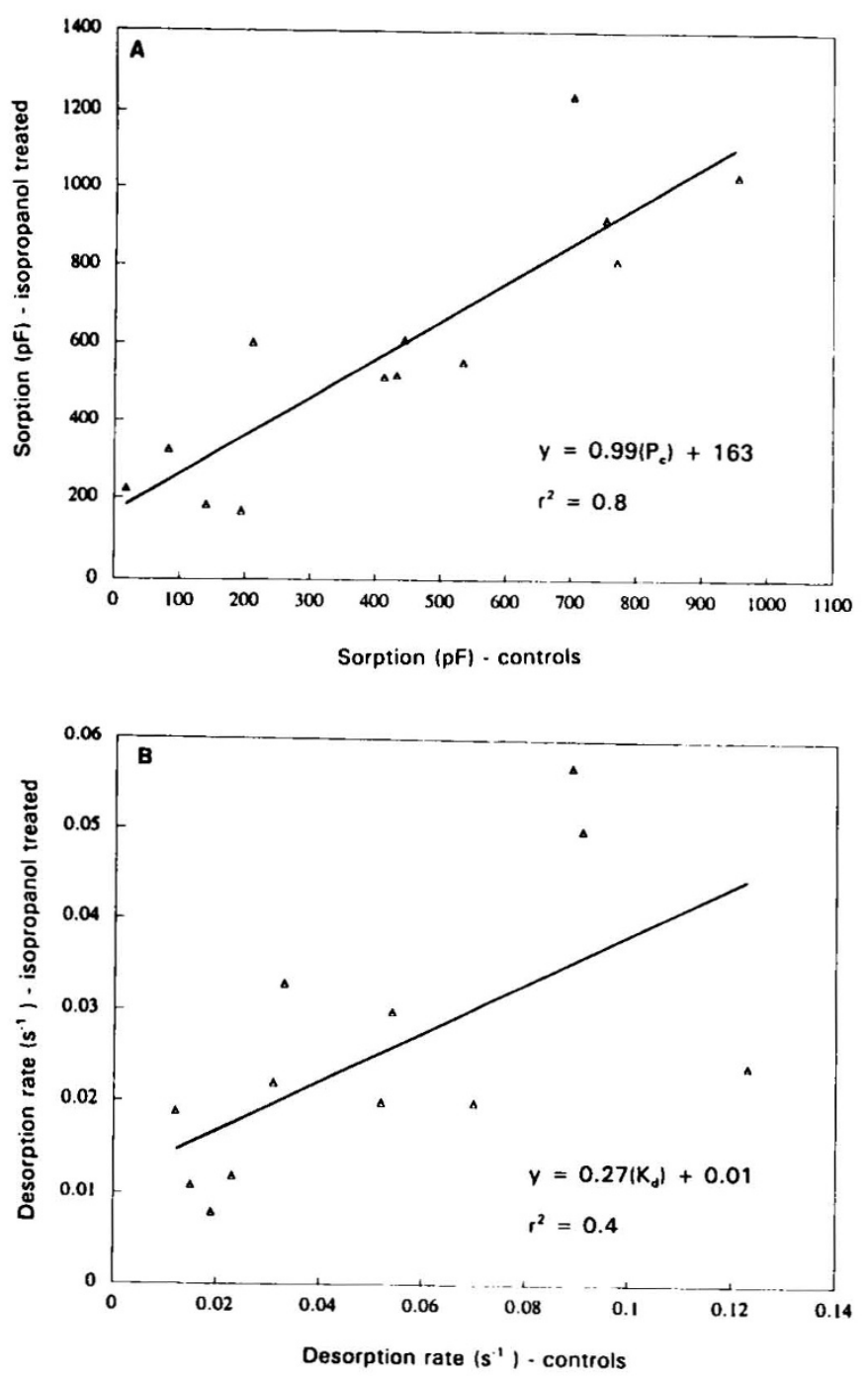

Fig. 4. A. Plot of maximum water sorption at paired control and isopropanol-treated sites $(\Delta)$ on the chest of 13 term infants after loading with water. The equation for the line of best fit is shown. Linear regression analysis shows positive correlation between paired measurements $\left(r^{2}=\right.$ $0.8, p<0.001)$. B. Plot of desorption rates of paired control and isopropanol-treated sites $(\Delta)$ on the chest of 13 healthy term infants after loading with water. Desorption rates were determined by 1 st-order kinetic analysis. The equation for the line of best fit is shown. Linear regression analysis shows a positive correlation between paired measurements. $r^{2}=$ $0.4, p<0.05$ )

urements have been used to study human skin $(6-8,11-15)$. During the last trimester, for example, the production of vernix in conjunction with development of the stratum corneum markedly changes the surface electrical properties of the fetus $(16,17)$. The vernix results in the formation of a high resistance "envelope" with subsequent disappearance of the fetal ECG as recorded from the surface of the maternal abdomen. In the newborn period, the skin surface has been studied using electrical conductance as a measure of surface hydration (7). Capacitance, a closely related electrical measurement, has been used extensively in the study of the skin of adult humans and animals $(6,8,13$, 14). The evaporimeter, a commonly used instrument in studies of transepidermal water loss, measures capacitance changes in polymer films as a function of humidity at various distances from the skin surface (18).

The findings in the present study are consistent with the recent report of "dry skin" in the human newborn by Saijo and Tagami (7), who used electrical conductance measurements as a measure of surface hydration. These investigators observed that the skin 
of term newborns in the 1st wk of life sorbs less water than that of their mothers over comparable body sites. Such a finding is consistent with a more hydrophobic skin surface in the infant than in the adult but does not necessarily indicate differences in stratum corneum water-holding properties.

A comparison of their values using conductance (7) and the values in our study using capacitance reveals similar variability in both the baseline measurements and measurements after water loading (sorption). This variability may be attributable to normal biologic differences in the skin surface among individuals, or it may be that the degree of water sorption is a direct function of the quantity of surface lipids present at the measurement site. In support of the latter interpretation, isopropanol treatment resulted in the highest sorption values in those individuals with the highest control sorption values (Fig. $4 A$ ). Conversely, infants with low water sorption at control sites had low water sorption at isopropanol-treated sites. Desorption rates showed a trend similar to sorption but were less well correlated (Fig. 4B). These findings are consistent with the hypothesis that more hydrophobic skin surfaces have higher amounts of surface lipids, which are incompletely removed by the method of solvent treatment used in the study. Thus, the observed variation in the control group might be attributable to differences in the amount of surface lipids.

In the discussion of these findings, it is important to recognize that sorption-desorption and transepidermal water loss are different biologic phenomena. Both phenomena are characterized by an interaction between water and skin. The former, however, relates to the transient interaction of the skin surface with exogenous water, whereas the latter generally refers to the movement of endogenous water across the skin. In both events, skin surface lipids may play a limiting role. Our study shows that sorption of water on the skin surface increases significantly after pertubation of the surface by isopropanol. Other investigators have shown that transepidermal water loss decreases significantly in premature infants after the application of artificial lipids to the skin surface (19). Thus, a superficial lipid layer such as the vernix could in fact act as both a protective barrier against excessive sorption of exogenous water onto the skin surface and a protective barrier against endogenous water loss, aiding in postnatal hydration and plasticization of the underlying stratum corneum.

In summary, we propose that the ability of the newborn skin to limit sorption and increase the desorption rate of topically applied water is an important physicochemical feature that may contribute to the successful transition from a warm, aqueous intrauterine milieu to a cool, dry extrauterine environment. We speculate that the presence of a superficial lipid layer enclosing the newborn infant may minimize the amount of heat lost by evaporation of amniotic fluid from the skin. Theoretically, the amount of heat lost by evaporation is dependent on the volume of water; thus, the limitation of sorption may be the parameter of greater physiologic significance. Such a mechanism would be most advantageous during unassisted births. It is conceivable therefore that a hydrophobic skin surface developed in hairless newborn mammals in part as a mechanism for thermoregulatory adaptation at birth.

Acknowledgments. The authors thank Reiko Tanaka and William L. Pickens for their technical assistance and Reginald Tsang, M.D., for helpful comments at various stages of the execution of this study.

\section{REFERENCES}

1. Strauss JS, Downing DT, Ebling FJ, Stewart ME 1991 Sebaceous glands. In: Goldsmith LA (ed) Physiology, Biochemistry, and Molecular Biology of the Skin. Oxford University Press, New York, pp 712-740

2. Williams ML. Hincenbergs M, Holbrook KA 1988 Skin lipid content during early fetal development. J Invest Dermatol 91:263-268

3. Williams ML 1992 Composition of skin lipids. In: Polin R, Fox W (eds) Fetal and Neonatal Physiology. WB Saunders Co, Philadelphia, pp 551-552

4. Downing DT, Stewart ME, Wertz PW, Colton SW, Abraham W, Strauss JS 1987 Skin lipids: an update. J Invest Dermatol 88(suppl):2S-6S

5. Chameides L, The AHA/AAP Neonatal Resuscitation Steering Committee 1990 Textbook of Neonatal Resuscitation. American Heart Association and American Academy of Pediatrics, Elk Grove Village, IL. pp 2-9

6. Wickett RR, Mutschelknaus JL, Hoath SB 1993 Ontogeny of water sorptiondesorption in the perinatal rat. J Invest Dermatol 100:407-411

7. Saijo S, Tagami H 1991 Dry skin of newborn infants: functional analysis of the stratum corneum. Pediatr Dermatol 8:155-159

8. Jemec GB, Serup J 1990 Epidermal hydration and skin mechanics. The relationship between electrical capacitance and the mechanical properties of human skin in vivo. Acta Derm Venereol (Stockh) 70:245-247

9. Wysocki SJ, Grauaug A, O'Neill G. Hahnel R 1981 Lipids in forehead vernix from newborn infants. Biol Neonate 39:300-304

10. Ramasastry P, Downing DT, Pochi PE, Strauss JS 1970 Chemical composition of human skin surface lipids from birth to puberty. J Invest Dermatol 54:139-144

11. Serup J 1992 A three-hour test for rapid comparison of effects of moisturizers and active constituents (urea). Measurement of hydration, scaling and skin surface lipidization by noninvasive techniques. Acta Derm Venereol Suppl (Stockh) 177:29-33

12. Blichmann CW, Serup J. Winther A 1989 Effects of single application of a moisturizer: evaporation of emulsion water, skin surface temperature, electrical conductance, electrical capacitance, and skin surface (emulsion) lipids. Acta Derm Venereol Suppl (Stockh) 69:327-330

13. Muramatsu K, Hirose S. Yukitake K, Ogata H, Mitsudome A. Oda T 1986 Relationship between maturation of the skin and electrical skin resistance. Pediatr Res 21:21-24

14. Emery MM, Hebert AA, Vila-Coro AA, Prager TC 1991 The relationship between skin maturation and electrical skin impedance. J Dermatol Sci 2:336-340

15. Tagami H 1980 Evaluation of skin surface hydration in vivo by electrical measurement. J Invest Dermatol 75:500-507

16. Oostendorp TF, van Oosterom A, Jongsma HW 1989 Electrical properties of tissues involved in the conduction of foetal ECG. Med Biol Eng Comput 27:322-324

17. Oostendorp TF, van Oosterom A, Jongsma HW 1989 The effect of changes in the conductive medium on the fetal ECG throughout gestation. Clin Phys Physiol Meas 10(suppl B): 11-20

18. Pinnagoda J, Tupker RA, Agner T, Serup J 1990 Guidelines for transepidermal water loss (TEWL) measurement. A report from the Standardization Group of the European Society of Contact Dermatitis. Contact Dermatitis 22:164178

19. Rutter M. Hull D 1981 Reduction of skin water loss in the newborn. I. Effect of applying topical agents. Arch Dis Child 56:669-672 